\title{
Evolution Equations for Di-hadron Fragmentation Functions
}

\author{
Marco Radici \\ INFN and Dipartimento di Fisica Nucleare e Teorica - Università di Pavia \\ via Bassi 6, I27100 Pavia - Italy
}

\begin{abstract}
Di-hadron Fragmentation Functions describe the probability that a quark hadronizes into two hadrons plus anything else, i.e. the process $q \rightarrow h_{1} h_{2} X$. Via a suitable singlespin asymmetry in semi-inclusive deep inelastic scattering (SIDIS), they can be used to extract the quark transversity distribution $h_{1}$ in the nucleon, a missing cornerstone of the nucleon partonic spin structure. I will discuss their evolution equations when they are explicitly depending on the invariant mass of the two hadrons. The equations are necessary to connect two-particle-inclusive measurements at different energies.
\end{abstract}

\section{Introduction}

Di-hadron Fragmentation Functions (DiFF) have been introduced for the first time in the context of $e^{+} e^{-} \rightarrow h_{1} h_{2} X$ reaction [2], and later have been recognized to be necessary in order to guarantee factorization of all collinear singularities [3]. However, in all these studies DiFF were always considered as functions only of the energy fractions $z_{1}$ and $z_{2}$ delivered to the two hadrons, while most of the experimental information consists of their invariant mass distribution $M_{h}$. Also the twist analysis of the quarkquark correlator for two-hadron inclusive production (see Fig. 1) indicates that the extracted DiFF are in general functions also of the pair relative momentum $R=P_{1}-P_{2}$ [4], whose transverse spatial component

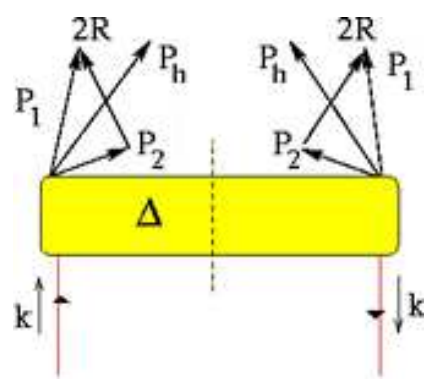

Figure 1: The quark-quark correlator for fragmentation in two hadrons.

$\mathbf{R}_{T}$ is related to $M_{h}$ [5]. In this case, I will refer to the socalled extended DiFF (extDiFF).

ExtDiFF can act as spin analyzers of the fragmenting quark; in particular the transverse polarization $\mathbf{s}_{T}$ of the latter can be related to the azimuthal orientation of the plane containing $\mathbf{P}_{1}$ and $\mathbf{P}_{2}$ via the mixed product $\mathbf{s}_{T} \cdot \mathbf{P}_{1} \times$ $\mathbf{P}_{2}$ (see Fig. 2). The strength of this effect is described by the extDiFF $H_{1}^{\varangle}$. In SIDIS on transversely polarized targets, this function appears in combina-

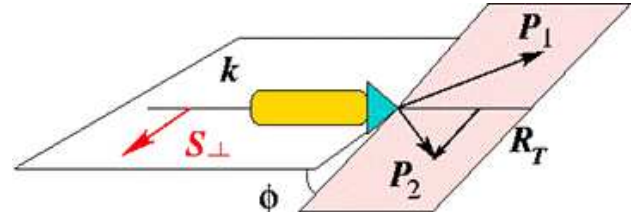

Figure 2: The nonperturbative effect $\mathbf{s}_{T} \cdot \mathbf{P}_{1} \times \mathbf{P}_{2}$ generating the single-spin asymmetry. tion with the transversity function, a leading-twist partonic distribution yet undetermined. The unknown extDiFF can be extracted from $e^{+} e^{-}$annihilations in two hadron pairs [6].

The HERMES and COMPASS collaborations have recently reported preliminary measurements of the asymmetry induced by the $\mathbf{s}_{T} \cdot \mathbf{P}_{1} \times \mathbf{P}_{2}$ effect at the average scale $\left\langle Q^{2}\right\rangle=2.53$ 
$\mathrm{GeV}^{2}[7,8]$. The BELLE collaboration is planning to extract $H_{1}^{\varangle}$ in $e^{+} e^{-}$annihilation but at the higher scale $s \approx 100 \mathrm{GeV}^{2}[9]$. In this talk I will discuss the evolution equations for extDiFF.

\section{Evolution equations for DiFF}

As already anticipated in Sec. $1, \mathrm{DiFF}$ are necessary to get a finite cross section for the $e^{+} e^{-} \rightarrow h_{1} h_{2} X$ process at NLO order [3]. The reason relies in the indistinguishability of the two mechanisms depicted in Fig. 3, which both lead to the observed hadron pair, either through $\mathrm{DiFF}$ or through separate single-hadron fragmentations after a partonic branching occurring at an arbitrary scale, intermediate between the hard $Q^{2}$ one and the soft $Q_{0}^{2}$ one. The consequence is the appearance of an inhomogenous term in the evolution equations for $\operatorname{DiFF}[3]$.
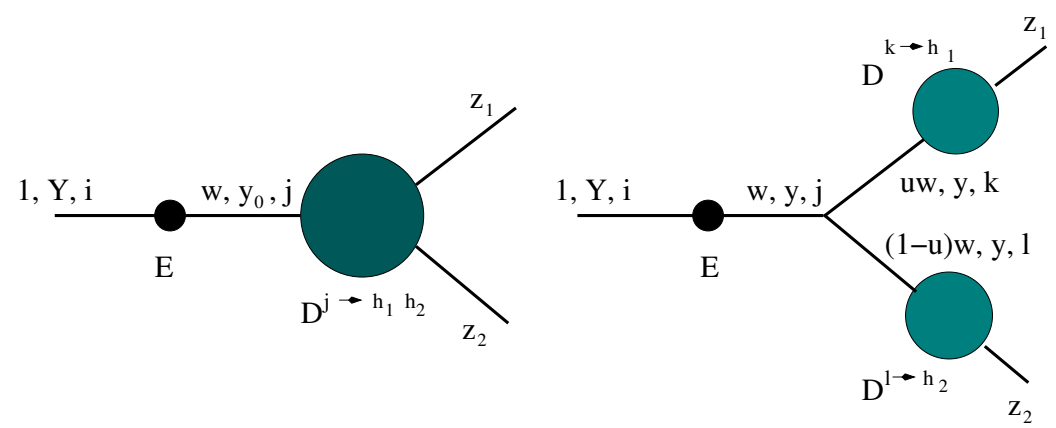

Figure 3: Double- and single-hadron fragmentations; the momentum fractions are indicated along with the scales and the parton indices; the black dots represent the parton evolution function $E$ (see text).

Making use of the techniques of jet calculus [2], the result of Ref. [3] can be easily reproduced when the two hadrons are emitted close in phase space (i.e., inside the same jet) and wide-angle hard partons are neglected. The phase-space structure of collinear singularities singled out in the fixed-order calculation of Ref. [3] can be translated in jet calculus as a degeneracy in all possible competing mechanisms that could realize the desired final state.

It is convenient to introduce the evolution variable

$$
y=\frac{1}{2 \pi \beta} \ln \left[\frac{\alpha_{s}\left(Q_{0}^{2}\right)}{\alpha_{s}\left(Q^{2}\right)}\right],
$$

between some two arbitrary hard $Q^{2}$ and soft $Q_{0}^{2}$ scales. If working at Leading Log Approximation (LLA), $\alpha_{s}$ and $\beta$ are the usual strong coupling constant and $\beta$ function, both at one loop. We can introduce also the parton evolution function $E_{j}^{i}(x, y)$, which expresses the probability of finding a parton $j$ at scale $Q_{0}^{2}$ with a momentum fraction $x$ of the parent parton $i$ at scale $Q^{2}$. It satisfies standard DGLAP evolution equations [2] and can be shown to resum all collinear leading logarithms of the kind $\alpha_{s}^{n} \ln ^{n}\left(Q^{2} / Q_{0}^{2}\right)$ [10]. The evolution variable $Y$ corresponding to the initial hard scale $Q^{2}$ is not zero, as one could deduce from Eq. 1, but can be defined by replacing $Q_{0}^{2}$ with the renormalization scale $\mu_{R}^{2}$ in Eq. 1 itself. 
In this picture, the fragmentation process of Fig. 3 is described by

$$
\begin{aligned}
\frac{1}{\sigma_{\mathrm{jet}}} \frac{d \sigma^{i \rightarrow h_{1} h_{2}}}{d z_{1} d z_{2} d Y}=\int_{z_{1}+z_{2}}^{1} \frac{d w}{w^{2}} E_{j}^{i}(w, Y & \left.-y_{0}\right) D^{j \rightarrow h_{1} h_{2}}\left(\frac{z_{1}}{w}, \frac{z_{2}}{w}, y_{0}\right) \\
+\int_{y_{0}}^{Y} d y \int_{z_{1}+z_{2}}^{1} \frac{d w}{w^{2}} & \int_{\frac{z_{1}}{w}}^{1-\frac{z_{2}}{w}} \frac{d u}{u(1-u)} E_{j}^{i}(w, Y-y) \hat{P}_{k l}^{j}(u) \\
& \times D^{k \rightarrow h_{1}}\left(\frac{z_{1}}{w u}, y\right) D^{l \rightarrow h_{2}}\left(\frac{z_{2}}{w(1-u)}, y\right)
\end{aligned}
$$

where $\hat{P}$ are the usual real Altarelli-Parisi splitting functions, $D^{i \rightarrow h}$ are single-hadron fragmentation functions, and $D^{i \rightarrow h_{1} h_{2}}$ are DiFF. Taking the derivative $d / d Y$ of Eq. 2, and further transforming the dependence upon $Y$ back to the one on $Q^{2}$, it easy to recover the inhomogeneous evolution equation for $D^{i \rightarrow h_{1} h_{2}}$ in the jet calculus language [10].

\section{Evolution equations for extDiFF}

The difference between extDiFF and DiFF is the explicit dependence of the former upon the transverse component of the hadron pair relative momentum, $\mathbf{R}_{T}$, or, equivalently, upon their invariant mass $M_{h}$ through the relation $[5,10]$

$$
R_{T}^{2}=\frac{z_{1} z_{2}}{z_{1}+z_{2}}\left[\frac{M_{h}^{2}}{z_{1}+z_{2}}-\frac{M_{1}^{2}}{z_{1}}-\frac{M_{2}^{2}}{z_{2}}\right]
$$

Knowledge of the $R_{T}^{2}$ scale makes the scale of the partonic branching no longer arbitrary. In fact, at LLA the virtualities of the involved partons are related by [10]

$$
k_{j}^{2}=\frac{k_{k}^{2}}{u}+\frac{k_{l}^{2}}{1-u}+\frac{r_{T}^{2}}{u(1-u)} \approx r_{T}^{2} \approx R_{T}^{2},
$$

where $r_{T}^{2}$ is the relative momentum of the partons $k$ and $l$ carrying momentum fractions $u$ and $1-u$ of the parent parton $j$, respectively.

Consequently, the arbitrary intermediate scale $y$ appearing in the second term of Eq. 2 collapses to $y_{T}$, defined similarly to $Y$ but with the replacement $Q^{2} \leftrightarrow R_{T}^{2}$. The analogue of Eq. 2 for extDiFF becomes, therefore,

$$
\begin{array}{r}
\frac{1}{\sigma_{\text {jet }}} \frac{d \sigma^{i \rightarrow h_{1} h_{2}}}{d z_{1} d z_{2} d R_{T}^{2} d Y}=\int_{z_{1}+z_{2}}^{1} \frac{d w}{w^{2}} E_{j}^{i}\left(w, Y-y_{0}\right) D^{j \rightarrow h_{1} h_{2}}\left(\frac{z_{1}}{w}, \frac{z_{2}}{w}, R_{T}^{2}, y_{0}\right) \\
+\frac{\alpha_{s}\left(R_{T}^{2}\right)}{2 \pi R_{T}^{2}} \int_{z_{1}+z_{2}}^{1} \frac{d w}{w^{2}} \int_{\frac{z_{1}}{w}}^{1-\frac{z_{2}}{w}} \frac{d u}{u(1-u)} E_{j}^{i}\left(w, Y-y_{T}\right) \hat{P}_{k l}^{j}(u) \\
\times D^{k \rightarrow h_{1}}\left(\frac{z_{1}}{w u}, y_{T}\right) D^{l \rightarrow h_{2}}\left(\frac{z_{2}}{w(1-u)}, y_{T}\right) .
\end{array}
$$

Taking the derivative $d / d Y$ of the previous expression, and further transforming back to the usual $Q^{2}$, we finally get [10]

$$
\frac{d}{d \ln Q^{2}} D^{i \rightarrow h_{1} h_{2}}\left(z_{1}, z_{2}, R_{T}^{2}, Q^{2}\right)=\frac{\alpha_{s}\left(Q^{2}\right)}{2 \pi} \int_{z_{1}+z_{2}}^{1} \frac{d u}{u^{2}} D^{j \rightarrow h_{1} h_{2}}\left(\frac{z_{1}}{u}, \frac{z_{2}}{u}, R_{T}^{2}, Q^{2}\right) P_{j i}(u),
$$


where $P_{j i}$ are the complete Altarelli-Parisi splitting functions, including the virtual contributions.

The same result holds also for the polarized fragmentation function $H_{1}^{\varangle}$, provided that the splitting kernels $\delta P$ for transversely polarized partons are used [10]. Equation 6 can also be conveniently diagonalized using a double Mellin transformation [10].

On the basis of Eq. 6, we argue that the cross section at NLO order for the inclusive production of two hadrons $h_{1}$ and $h_{2}$, inside the same jet and with invariant mass $M_{h}$, can be expressed in the factorized form

$$
\frac{d \sigma^{h_{1} h_{2}}}{d z_{1} d z_{2} d R_{T}^{2} d Q^{2}}=\sum_{i} \sigma^{i} \otimes D^{i \rightarrow h_{1} h_{2}}\left(R_{T}^{2}, Q^{2}\right)
$$

where $\sigma^{i}$ are the same coefficient functions found in the case of single-hadron inclusive production.

\section{Acknowledgments}

I warmly thank F.A. Ceccopieri and A. Bacchetta, without whom this work would not have been possible.

\section{References}

[1] Slides:

http://indico. cern. ch/contributionDisplay.py?contribId=167\&sessionId=4\&conf Id=9499

[2] K. Konishi, A. Ukawa and G. Veneziano, Phys. Lett. B78 243 (1978).

[3] D. de Florian and L. Vanni, Phys. Lett. B578 139 (2004).

[4] A. Bianconi et al., Phys. Rev. D62 034008 (2000).

[5] A. Bacchetta and M. Radici, Phys. Rev. D67 094002 (2003).

[6] D. Boer, R. Jakob and M. Radici, Phys. Rev. D67 094003 (2003).

[7] P.B. van der Nat (HERMES), arXiv:hep-ex/0512019 (2005).

[8] A. Martin (COMPASS), arXiv:hep-ex/0702002 (2007).

[9] K. Hasuko et al., AIP Conf. Proc. 675454 (2003).

[10] F.A. Ceccopieri, M. Radici and A. Bacchetta, Phys. Lett. B650 81 (2007). 